\title{
A Road Departure Decision Method Using State Diagrams and Statistical Hypothesis Tests in Map Matching
}

\author{
Toshiyuki AOKI ${ }^{\dagger}$, Mikio BANDO ${ }^{\dagger}$, Tomoaki HIRUTA ${ }^{\dagger}$, \\ Koichi KATO $^{\dagger \dagger}$, Akihiro KAWABATA ${ }^{\dagger \dagger}$, Zhixing LIU ${ }^{\dagger \dagger}$ \\ $\dagger \quad$ Hitachi Research Laboratory, Hitachi, Ltd. \\ 1-1, Omika-cho 7-chome, Hitachi-shi, Ibaraki, 319-1292 Japan \\ $+{ }^{+}$Core Engineering Development Management Department, Clarion Co., Ltd. \\ E-mail: toshiyuki.aoki.gh@hitachi.com
}

\begin{abstract}
Car navigation systems display the car's position on a link, which is a line representing a road in map datum, when the car is running on a road and display its estimated position when it is running in a parking area. Therefore, it is necessary for map matching to decide whether a car is running on a road. The errors of the car's estimated position and the link position cause the incorrect decision when the car is running in a parking area parallel to the road. In this paper, a road departure decision method using statistic hypothesis tests and state diagrams is proposed. The statistic hypothesis tests make decisions considering the previous errors, and the state diagrams change decision conditions in each state. This method can therefore decrease the number of incorrect decisions that are made. The performance of this method was experimentally evaluated using observation data collected with a car, and the situation that this method could correctly decide on road departure was shown.
\end{abstract}

\section{Introduction}

In car navigation systems, locating a car consists of position estimation and map matching. The former estimates the position, velocity, heading, and their estimation error covariance from the output of a GPS receiver and sensors [1], [2]. The latter calculates the position and heading of link candidates near the estimated position on links and selects the most adequate link candidate. Map matching decides whether a car pulls off a road and sets the estimated position and heading to the position and heading of a car mark in the case of running on a road and the position and heading of the link candidate to them in the case of running outside a road, for example, running in a parking area. Previous map matching methods have been proposed that select the most adequate link candidate with various parameters that index the probability that the car is on the road [3]-[5]. However, methods for deciding road departure have rarely been proposed. If road departure decision methods simply use the same parameters as map matching methods, they sometime mistakenly judge that a car is run- ning on a road when it is actually running in a parking area parallel to a road. Furthermore, when the accuracy of estimations and links is reduced and a car is running on a road, the road departure decision methods mistakenly judge a car to be running outside a road. In this paper, we propose a road departure decision method using state diagrams and statistical hypothesis tests. This method calculates test statistics from errors of estimations and links, which are assumed to be normally distributed, and decides road departure by applying statistical hypothesis tests. Decision conditions differ depending on whether a car is running on a road or in a parking area, so this method decreases wrong decisions even when the accuracy of estimations and links is reduced and when the car is running in a parking area parallel to the road.

This paper is organized as follows. The proposed road departure decision method using state diagrams and statistical hypothesis tests is presented in Section 2. This method is performed and evaluated using observation data collected with a car in Section 3. Finally, we conclude the paper in Section 4.

\section{A Road Departure Decision Method}

The following conditions are satisfied when a car runs on roads. The third condition is added because it is possible to correctly decide outside-road running when a car runs in an underground parking area under a road.

Condition 1: A car's estimated position and link position do not differ.

Condition 2: A car's estimated heading and link direction do not differ.

Condition 3: A car is not running in an underground parking area.

The proposed method decides whether these conditions are correct and judges whether a car is running on a road on the basis of these results. 


\subsection{Test of Position Difference between Car and Road}

It is assumed that errors regarding the car's estimated position and the link position are independently and normally distributed. We consider the following test statistic $T_{p}$ :

$$
\begin{gathered}
T_{p}=\left[\begin{array}{ll}
\Delta x & \Delta y
\end{array}\right] \Sigma_{p}^{-1}\left[\begin{array}{l}
\Delta x \\
\Delta y
\end{array}\right] \\
{\left[\begin{array}{l}
\Delta x \\
\Delta y
\end{array}\right]=\left[\begin{array}{c}
x_{e} \\
y_{e}
\end{array}\right]-\left[\begin{array}{l}
x_{l r} \\
y_{l r}
\end{array}\right]} \\
\Sigma_{p}=\Sigma_{p e}+\Sigma_{p l} \\
\Sigma_{p l}=\left[\begin{array}{cc}
\sigma_{p l}^{2} \sin ^{2} \theta_{l} & 0 \\
0 & \sigma_{p l}^{2} \cos ^{2} \theta_{l}
\end{array}\right]
\end{gathered}
$$

where $\left(x_{e}, y_{e}\right)$ is the car's estimated position, $\left(x_{l r}, y_{l r}\right)$ is the position of the point near estimated position in the running region, the running region is the area between the ends of lanes (as shown in Fig. 1), $\theta_{l}$ is the link candidate heading, $\Sigma_{e p}$ and $\Sigma_{l p}$ are respectively the error covariance of the estimated position and the link candidate position, and $\sigma_{p l}^{2}$ is the error variance of the link candidate position. The car's estimated position and its covariance are obtained from the position estimation. In Fig. 1, a white-colored circles is the point near estimated position in the running region, a black-colored circle is a node which is a point on each side of the link, a black-colored line between nodes is the link, and a black-colored triangle is the car's estimated position.

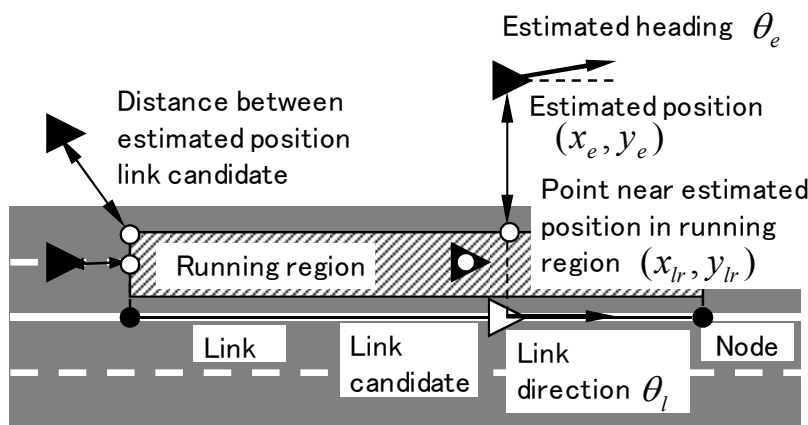

Fig. 1: Distance between car's estimated position and link candidate

The test statistic $T_{p}$ indicates the difference between the car's estimated position and the position of the link candidate. It is chi-squared distributed with 1 degree of freedom from the previous assumption. Therefore, the proposed method decides whether their positions differ by applying a chi-squared test. The probability that a car is on a road corresponds to the value of the integral according to the probability density function of a chi-squared distribu- tion when the test statistic $T_{p}$ is larger than the calculated value, as shown in Fig. 2.

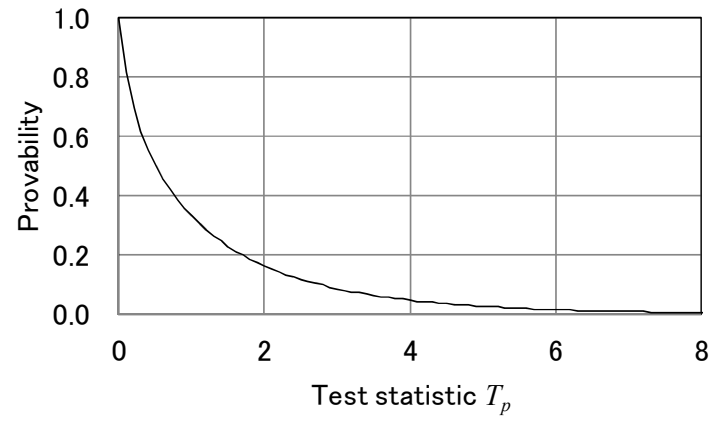

Fig. 2: Probability that a car is on a road

Two hypotheses are thus formulated:

Null hypothesis $\boldsymbol{H}_{\boldsymbol{0}}$ : The car's estimated position and the link candidate position do not differ.

Alternative hypothesis $\boldsymbol{H}_{1 p}$ : The car's estimated position and the link candidate position differ.

This decision is based on a chi-squared test. That is, the significance level $\alpha$ is set, and the hypotheses are accepted according to the following conditions:
If $T_{p} \leq \chi_{\alpha}^{2}(1)$, accept null hypothesis $H_{0 p}$.
If $T_{p}>\chi_{\alpha}^{2}(1)$, accept alternative hypothesis $H_{l p}$.

\subsection{Test of Heading Difference Between Car and Road}

It is assumed that errors regarding the car's estimated heading and the link direction are independently and normally distributed. We consider the following test statistic $T_{\theta}$ :

$$
\begin{gathered}
T_{\theta}=\frac{\Delta \theta}{\sigma_{\theta}} \\
\Delta \theta=\theta_{e}-\theta_{l} \\
\sigma_{\theta}^{2}=\sigma_{\theta e}^{2}+\sigma_{\theta l}^{2}
\end{gathered}
$$

where $\theta_{e}$ and $\sigma_{\theta e}^{2}$ are the car's estimated heading and its variance, respectively, and $\sigma_{\theta l}^{2}$ is the variance of the link candidate heading.

The test statistic $T_{\theta}$ indicates the difference between the car's estimated heading and the link candidate position. It is normally distributed from the previous assumption. Therefore, the method decides whether their headings differ by applying a statistic hypothesis test.

Two hypotheses are thus formulated 
Null hypothesis $\boldsymbol{H}_{\boldsymbol{\theta} \boldsymbol{\theta}}$ : The car's estimated heading and the link candidate heading do not differ.

Alternative hypothesis $\boldsymbol{H}_{1 \theta}$ : The car's estimated heading and the link candidate heading differ.

This decision is based on a statistic hypothesis test. That is, the significance level $\alpha$ is set, and the hypotheses are accepted according to the following conditions:

$$
\begin{aligned}
& \text { If }\left|T_{\theta}\right| \leq N_{\alpha / 2} \text {, accept null hypothesis } H_{0 \theta} \text {. } \\
& \text { If }\left|T_{\theta}\right|>N_{\alpha / 2} \text {, accept alternative hypothesis } H_{1 \theta} \text {. }
\end{aligned}
$$

\subsection{In-underground-parking-area Running De- cision}

When a car enters a underground parking area, the distance between a car and a road increases and a GPS receiver does not receive any signals from GPS satellites. When the car exits the underground parking area, the distance between the car and the road which is not a tunnel and a GPS receiver can receive some signals. The proposed method utilizes both phenomena and decides whether a car is running in an underground parking area by applying the state diagram shown in Fig. 3. The state of in-underground-parking-area running and the state of outside-underground-parking-area running are set and the following decision conditions are used:

Condition 4: The number of GPS signals in a receiver is zero.
Condition 5: The distance between the estimated position and the link candidate is longer than $15 \mathrm{~m}$.

Condition 6: The link attribute of the link candidate indicates a tunnel

In the state of in-underground-parking-area running, the state changes to the state of outside-underground-parking-area running if Conditions 4 and 5 are satisfied. If they are not, the method decides in-underground-parking-area running. In the state of outside-underground-parking-area running, the state changes to the state of in-underground-parking-area running if Condition 4 is not satisfied or if Conditions 5 and 6 are not satisfied. Otherwise, the method decides on outside-underground-parking-area running.

By using this state diagram, the method can correctly decide whether a car is running in a underground parking area under a road.

\subsection{Road Departure Decision}

The method decides whether Conditions 1-3 are satisfied, using the procedures described in Paragraphs 2.1-2.3, and then decides on road departure on the basis of the results. The state diagram shown in Fig. 4 is applied to the method. The states of around-road running and in-parking-area running are set. In the state of around-road running, the state changes to the state of in-parking-area running if Condition 1 or 3 is not satisfied. The method decides on on-road running if Conditions 1-3 are satisfied and decides on outside-road running if not. In the state of

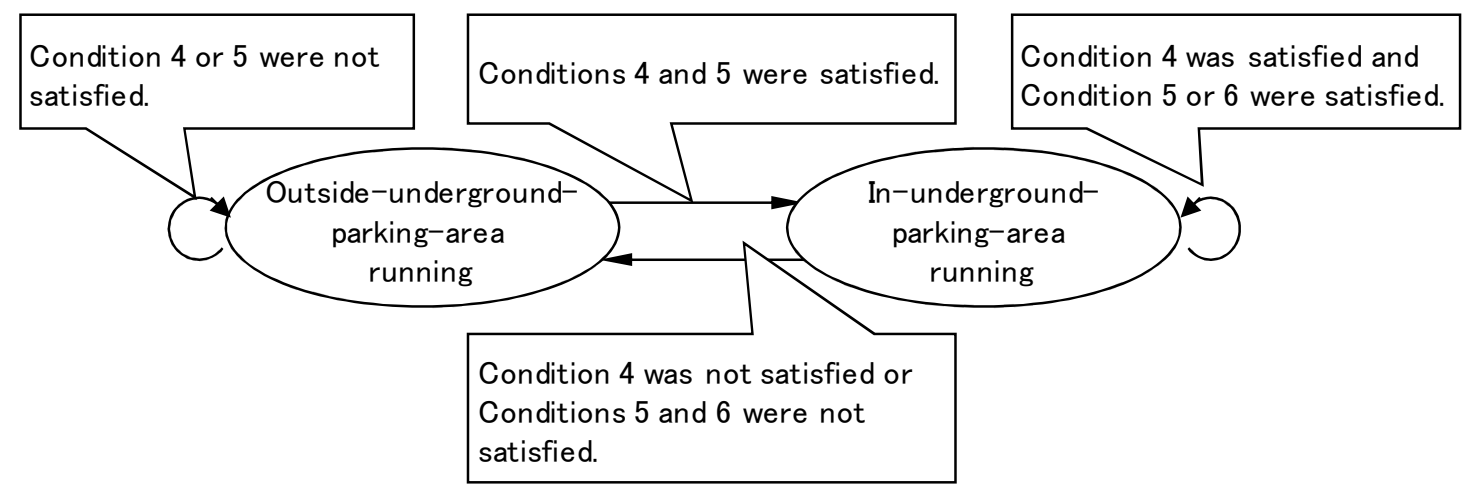

Fig. 3: State diagram of in-underground-parking-area running decision

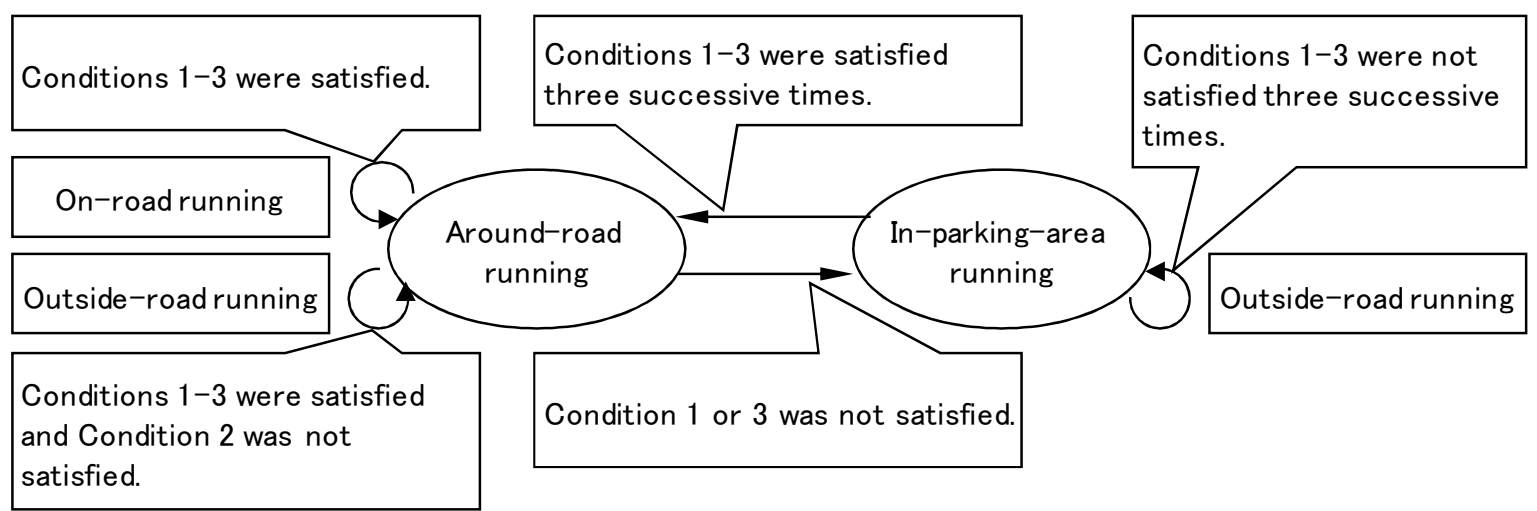

Fig. 4: State diagram of road departure decision 
in-parking-area running, the state changes to the state of around-road running if Conditions 1-3 are satisfied three successive times. If not, the method decides on outside-road running. It is possible to prevent a single incorrect decision because the state changes when conditions are satisfied three successive times.

\section{Experimental Evaluation}

\subsection{Experiments of Observation Data Collection}

A GPS receiver, odometer, gyro and accelerator were installed in a car. The accelerator measured acceleration in the up-and-down direction in the car. The gyro measured angular velocity in the revolution direction. The outputs of the GPS receiver and the sensors were recorded in a memory device in 1 and $10 \mathrm{~Hz}$, respectively, from 15:54 to 16:00 on October 7, 2008. The car ran in a parking area parallel to a road which located in Yamato City, Kanagawa, Japan. The running patterns are shown in Fig. 5. The car ran twice in pattern 1 and once in pattern 2 .

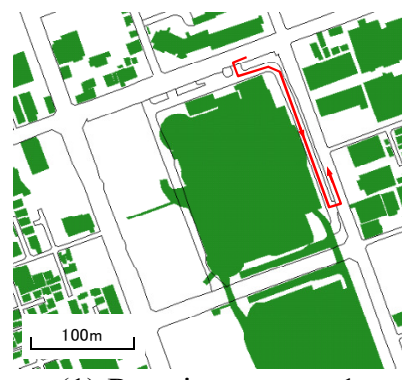

(1) Running pattern 1

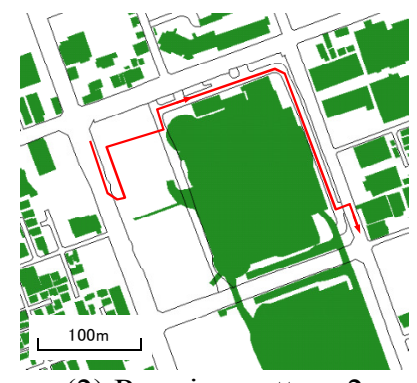

(2) Running pattern 2

Fig. 5: Running patterns

(Note: The above figure utilizes the basic map information provided by the Geospatial Information Authority of Japan.)

\subsection{Evaluation Method and Results}

We used a personal computer to process the position estimation and map matching, including the road departure decision method described in Section 2, using the outputs of the GPS receiver and the sensors. The results of the position measurement were then plotted on a map (see Figs. 6 and 7). An incorrect decision occurred in the route that was about 15 meters from the road.

We evaluated the performance of the proposed method by determining the correct decision rate which was the number of correct decisions divided by the total number of

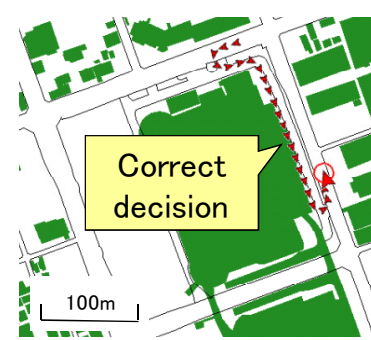

(1) Result of first try

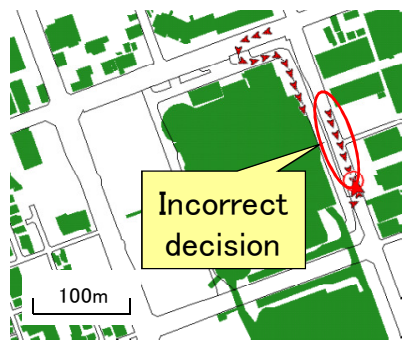

(2) Result of second try
Fig 6: Results of position measurement in Pattern 1

(Note: The above figure utilizes the basic map information provided by the Geospatial Information Authority of Japan.)

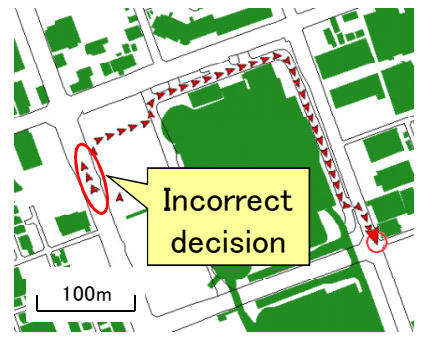

Fig 7: Results of position measurement in Pattern 2 (Note: The above figure utilizes the basic map information provided by the Geospatial Information Authority of Japan.)

decisions in a parking area twice in running pattern 1 and once in running pattern 2 (shown in Table 1). In the performance evaluation, we measured the length between the car's position and the road, and the difference between the car's heading and the road direction, plotting the estimated positions on air photographs, where it was assumed that the car ran in the center of the route in the parking area and the car's heading was the same as the estimated heading. The proposed method could correctly decide on road departure when the distance between the car's position and the road was more than 15 meters, and when the difference between the car's heading and the road direction was more than 15 degrees.

\section{Conclusions}

We proposed a road departure decision method using state diagrams and statistical hypothesis tests in map matching for car locating, to prevent from a single wrong decision and consider errors of position and heading of estimation and link. This method could correctly decide on road departure when the distance between the car's position and the road is more than 15 meters, and when the difference between the car's heading and the road direction was more than 15 degrees.

Table 1: Correct decision rate in a parking area

\begin{tabular}{|c|c|c|c|}
\hline $\begin{array}{lr}\begin{array}{l}\text { Difference } \\
\text { between car's } \\
\text { heading and road direction }\end{array} & \begin{array}{r}\text { Length between car's } \\
\text { position and road }\end{array} \\
\end{array}$ & Under 10 meters & $\begin{array}{l}\text { More than } 10 \text { meters } \\
\text { and under } 15 \text { meters }\end{array}$ & More than 15 meters \\
\hline Under 15 degrees & - & $73.8 \%(31 / 42)$ & $100.0 \%(20 / 20)$ \\
\hline More than 15 degrees & $100.0 \%(6 / 6)$ & $100.0 \%(3 / 3)$ & $100.0 \%(4 / 4)$ \\
\hline
\end{tabular}




\section{References}

[1] M. Bando, Y. Kawamata and T. Aoki: Dynamic Sensor Bias Correction for Attitude Estimation using Unscented Kalman Filter in Autonomous Vehicle, Proceedings of the 42th ISCIE International Symposium on Stochastic Systems Theory and Its Applications, pp. 33-39, Okayama, November 2010.

[2] M. Bando, Y. Kawamata, T. Aoki and K. Tanaka: The Study of Localization and Attitude Estimation for Autonomous Medium Speed Vehicle in an Outdoor Location, Proceedings of SICE 10th Annual Conference on Control Systems, 161-1-4, Kumamoto, March 2010.

[3] J. Pyo, D. Shin, and T. Sung: Development of a Map Matching method using the multiple hypothesis technique, 2001 IEEE Intelligent Transportation Systems Conference Proceedings, pp. 23-27, Oakland, August 2001.

[4] W. Kim, G. Jee, and J. Lee: Efficient Use of Digital Road Map in Various Positioning for ITS, IEEE Symposium on Position Location and Navigation, pp. 170-176, 2000.

[5] H. Morohashi, K. Abe, Y. Tsukamoto, K. Uehara, and H. Seino: A Map Matching Algorithm using Vehicle Existence Coefficient, The 25th Sensing Forum, pp. 268-271, Saga, November 2008. 


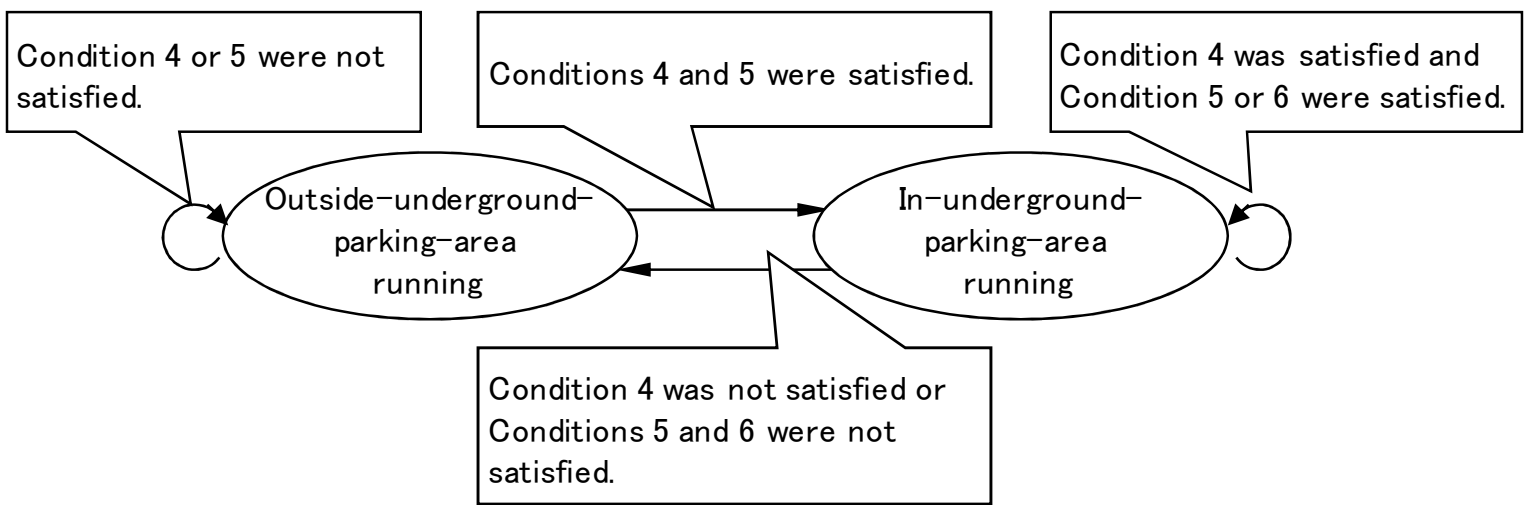

Fig. 3: State diagram of in-underground-parking-area running decision

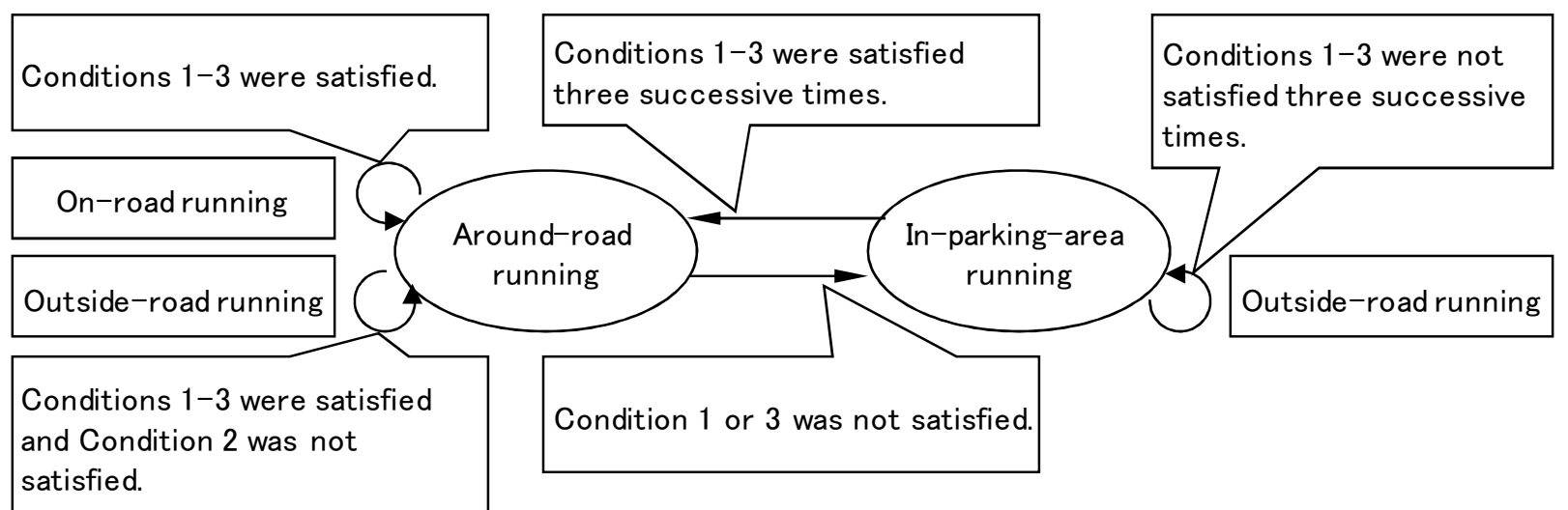

Fig. 4: State diagram of road departure decision

Table 1: Correct decision rate in a parking area

\begin{tabular}{|l|c|c|c|}
\hline $\begin{array}{r}\text { Length between car's } \\
\text { pifference } \\
\text { between car's andion and road } \\
\text { heading and road direction }\end{array}$ & Under 10 meters & $\begin{array}{c}\text { More than 10 meters } \\
\text { and under 15 meters }\end{array}$ & More than 15 meters \\
\hline Under 15 degrees & - & $73.8 \%(31 / 42)$ & $100.0 \%(20 / 20)$ \\
\hline More than 15 degrees & $100.0 \%(6 / 6)$ & $100.0 \%(3 / 3)$ & $100.0 \%(4 / 4)$ \\
\hline
\end{tabular}

